\title{
Erratum to: Requirements Engineering
}

\section{Erratum to:}

\section{J. Dick et al., Requirements Engineering}

https://doi.org/10.1007/978-3-319-61073-3

The published version of this book had incomplete affiliation for the author Elizabeth Hull. The affiliation have been corrected and the text has been updated in the book.

The updated online version of the book can be found at https://doi.org/10.1007/978-3-319-61073-3 